\title{
Effect of oral antibiotics on intestinal production of propionic acid
}

\author{
A F Mellon, S A Deshpande, J C Mathers, K Bartlett
}

\begin{abstract}
Background-Propionic acid derived from colonic bacterial fermentation contributes substantially to overall propionate load in children with disorders of propionate metabolism, and its reduction is important for adequate metabolic control.

Aims-To evaluate the in vitro and in vivo effects of antibiotic treatment on propionate production by colonic bacteria, and plasma propionate concentrations in a child with propionic acidaemia.

Methods-In vitro fermentation techniques were used to study the effects of addition of antibiotics (metronidazole, clindamycin, erythromycin, and vancomycin) on net faecal production of short chain fatty acids including propionic acid. Courses of oral antibiotics of 7 days duration were used to assess the in vivo effects on faecal propionate production and metabolic control including plasma propionate concentrations.
\end{abstract}

Results-Metronidazole produced the largest and most consistent reduction (77$84 \%$ ) in the production in vitro of propionate from faecal homogenates. Oral administration of metronidazole reduced faecal propionate production by $43 \%$ within 24 hours of treatment; a 7 day course virtually eliminated it for the next 3 weeks. These reductions were accompanied by substantially lowered plasma propionate concentrations during the same period.

Conclusions-Intermittent courses of oral metronidazole might be as effective as continuous treatment in reducing gut propionate production in children with disorders of propionate metabolism. (Arch Dis Child 2000;82:169-172)

Keywords: propionic acidaemia; antibiotics; fermentation

Propionic acidaemia is a life threatening inborn error of propionate metabolism, which usually presents in the neonatal period with vomiting, lethargy, hypoglycaemia, hyperammoniaemia, and ketoacidosis. Its treatment consists of minimising propionate load from all sources, promotion of detoxification and excretion of propionyl CoA through the use of L carnitine, and careful management during acute intercurrent illnesses. ${ }^{1}$ In addition to its formation during the catabolism of amino acids (isoleucine, valine, methionine, and threonine) and $\beta$ oxidation of fatty acids containing odd num- bers of carbon atoms, propionyl CoA is also derived from anaerobic bacterial fermentation of carbohydrates in the colon. ${ }^{2}$ The maximum contribution of catabolism of propiogenic amino acids to total propionate production has been assessed to be between $5 \%$ and $66 \%,{ }^{2}{ }^{3}$ with gut bacterial fermentation accounting for a large proportion of the rest. ${ }^{2}$ Therefore, effective reduction of the latter source is important for adequate metabolic control.

Using an in vitro fermentation technique, it has been shown that the addition of metronidazole greatly reduces the production of short chain fatty acids, including propionic acid. ${ }^{4}$ In a study of four children with propionic acidaemia, the administration of metronidazole resulted in substantial reductions in gut bacterial activity, as measured by changes in the lactulose breath hydrogen test and reduced faecal bacterial colony counts, as well as large reductions in stool propionate excretion. ${ }^{5}$ This observation has led to the suggestion that metronidazole should be used as an adjunct to other therapeutic manoeuvres in the management of propionic acidaemia.

On the other hand, Hoverstad et al, studying the effects of a number of orally administered antibiotics on short chain fatty acid excretion in healthy adult volunteers, found that metronidazole did not produce any alteration in faecal short chain fatty acid excretion and neither was it detectable in the faeces. ${ }^{6}$ However, nonabsorbable antibiotics such as clindamycin and vancomycin greatly reduced excretion of short chain fatty acids, including propionic acid. ${ }^{7}$ Accordingly, it was postulated that orally administered metronidazole was almost completely absorbed in the small intestine and was not secreted into the large bowel in sufficient concentrations to affect fermentation by anaerobic bacteria.

Our study was prompted by these discrepant findings and the implication that orally administered metronidazole might not have as great an effect on large bowel fermentation as non-absorbable antibiotics. Our study was designed to evaluate the in vitro effects of various antibiotics on propionate production by colonic bacteria derived from faecal specimens from a child with propionic acidaemia. In addition, the in vivo effects of orally administered antibiotics on metabolic control, short chain fatty acid excretion, and in vitro fermentative ability of faecal homogenates from the same child were investigated.

\section{Patient and methods}

A 3 year old boy had presented on the 2 nd day of life with feeding difficulties, vomiting, dehydration, lethargy, and seizures. He was found to 
be hypoglycaemic, hyperammoniaemic, severely ketoacidotic, and to have hyperglycinaemia. A raised urinary methylcitrate suggested the diagnosis of propionic acidaemia, and this was confirmed by greatly reduced $(<5 \%)$ propionyl CoA carboxylase activity in his cultured skin fibroblasts. He was managed with a restricted protein intake of $<1.5 \mathrm{~g} / \mathrm{kg} /$ day and supplemental carnitine $(200 \mathrm{mg} / \mathrm{kg} /$ day $)$ and biotin (20 mg/day). He was monitored daily for ketonuria and weekly for ammonia concentrations.

IN VITRO INCUBATION OF STOOL WITH

ANTIBIOTICS TO ASSESS THE EFFECTS ON SHORT

CHAIN FATTY ACID PRODUCTION

Before treatment with antibiotics was started, a fresh stool sample was collected from the child and transferred at $4^{\circ} \mathrm{C}$ to the laboratory for the fermentation study. Fermentations were carried out using a protocol similar to that described by Edwards et al. ${ }^{8}$ The sample was weighed and mixed (1/3.125 wt/vol) with an anaerobic phosphate buffer $\left(0.1 \mathrm{M} \mathrm{NaH}_{2} \mathrm{PO}_{4}\right.$ and $0.1 \mathrm{M} \mathrm{Na} \mathrm{NPO}_{4}$, adjusted to $\mathrm{pH} 6.5$ ), homogenised in a nitrogen atmosphere, and filtered through muslin to produce a faecal suspension to be used as inoculum. Arabinogalactan, a non-starch polysaccharide, and potato starch (Sigma-Aldrich Company, Dorset, UK), were used as sources of carbohydrate and included in the incubations at a concentration of $20 \mathrm{~g} /$ litre. Incubations were performed in the presence of metronidazole, clindamycin, erythromycin, and vancomycin at final concentrations of $4 \mathrm{~g} /$ litre. The samples, including a set of control samples without added antibiotics, were incubated for 24 hours at $37^{\circ} \mathrm{C}$ under nitrogen in an orbital shaker and then stored at $-20^{\circ} \mathrm{C}$ pending analysis. Aliquots of incubation fluid were deproteinised using metaphosphoric acid solution with 3-methyl valerate as internal standard, as described previously. ${ }^{9}$ The short chain fatty acids were separated and quantified using a $3 \mathrm{~m}$ glass column packed with $10 \%$ SP-1200-1\% phosphoric acid on 80/100 Chromosorb (Supelco Inc, Belliforte, Pennsylvania, USA) in a Pye Unicam PU4550 gas chromatograph.

The initial concentration of each short chain fatty acid in the homogenate before incubation was subtracted from the concentrations at the end of 24 hours to give a measure of the net production of short chain fatty acids in the presence or absence of each of the antibiotics. The percentage change in 24 hour short chain fatty acid production as a result of antibiotic treatment was determined by comparing this with production in a control sample incubated without the antibiotic. The within subject standard deviations $\left(\zeta_{\omega}\right)$ and repeatability (the difference between two measurements for the same subject for $95 \%$ pairs of observations, calculated as $2.77 \zeta_{\omega}$ ) for faecal propionate and short chain fatty acid assays were 0.33 and $0.91 \mathrm{mmol} /$ litre, and 0.89 and $2.47 \mathrm{mmol} /$ litre, respectively.
THE EFFECT OF ORAL ANTIBIOTICS ON IN VIVO METABOLIC CONTROL IN PROPIONIC ACIDAEMIA The aim of our study was to assess the effect of orally administered antibiotics (erythromycin, vancomycin, and metronidazole) on propionic acid production in the gut and on the indices of metabolic control of the same index patient.

A six week cycle was used for assessing the effect of each of the antibiotics. After completion of a seven day course of each antibiotic in standard dosage, there was a five week washout period before the next course of treatment started. During this period, the child's dietary protein intake was maintained at $1.5 \mathrm{~g} / \mathrm{kg} /$ day and his usual medications were continued unchanged.

During each six week cycle, the following were measured before and 24 hours after each antibiotic was started, and on days 8 and 21 (except plasma propionate) of the cycle.

(1) Faecal propionate production using the in vitro incubation technique with potato starch substrate as previously described but without the addition of any further antibiotics. Duplicate stool specimens were incubated for short chain fatty acid estimations.

(2) Plasma propionic acid concentrations. Propionic acid was extracted with ethanol from the plasma samples using the method of Remesey and Demigne. ${ }^{10} \mathrm{~A}$ capillary column was used to measure propionate concentration with $10 \mu \mathrm{mol} /$ litre of isobutyric acid as the internal standard. Recovery of added propionate was determined by measuring the propionate concentrations in a volunteer plasma sample spiked with known concentrations of propionate $(0,2.5,5$, and $10 \mu \mathrm{mol} /$ litre). The within subject coefficient of variation for the propionate assay in plasma $(n=6)$ was $8.3 \%$.

(3) Blood intermediary metabolites (glucose, lactate, pyruvate, alanine, $\beta$-hydroxybutyrate, acetoacetate, glycerol, and non-esterified fatty acids) were determined by enzymatic assays using a Cobas Bio fast centrifugal analyser as described previously, ${ }^{11}$ along with serum ammonia.

The study protocol was approved by the joint ethics committee of Newcastle Health Authority and the University of Newcastle.

\section{Results}

\section{IN VITRO STUDY}

Metronidazole produced the largest fall in propionate production with both arabinogalactan and potato starch as substrates $(77 \%$ and $84 \%$, respectively). Moreover, the change in total short chain fatty acid production $(-22 \%$ and $-51 \%$, respectively) was greatest with this antibiotic. The other antibiotics produced greater falls in total short chain fatty acid production when potato starch was used as the substrate rather than arabinogalactan (table 1).

IN VIVO STUDY

Metronidazole given orally produced a fall of $43 \%$ in the propionate production by faecal 
Table 1 Effect of added antibiotics on net and short chain fatty acid (SCFA) production from faecal homogenates in a child with propionate acidaemia using arabinogalactan and potato starch as substrates

\begin{tabular}{|c|c|c|c|c|}
\hline \multirow[b]{2}{*}{ Antibiotic } & \multicolumn{2}{|c|}{ Net propionate production } & \multicolumn{2}{|c|}{ Net total SCFA production } \\
\hline & Arabinogalactan & Potato starch & Arabinogalactan & Potato starch \\
\hline No antibiotic & 13.8 & 8.1 & 51.0 & 40.3 \\
\hline Clindamycin & 12.5 & 3.1 & 57.8 & 21.6 \\
\hline Erythromycin & 13.0 & 4.7 & 55.5 & 24.1 \\
\hline Metronidazole & 3.1 & 1.3 & 39.7 & 19.5 \\
\hline Vancomycin & 9.9 & 3.1 & 45.5 & 18.2 \\
\hline
\end{tabular}

Antibiotics were at a concentration of $4 \mathrm{~g} /$ litre.

Net propionate and SCFA production were measured as $\mathrm{mmol} / \mathrm{litre}$ incubation mixture/day.

Table 2 Net propionic acid and total short chain fatty acid (SCFA) production during antibiotic treatment after incubation of faecal homogenate for 24 hours with potato starch

\begin{tabular}{|c|c|c|c|c|c|c|}
\hline & \multicolumn{2}{|c|}{ Metronidazole } & \multicolumn{2}{|c|}{ Erythromycin } & \multicolumn{2}{|l|}{ Vancomycin } \\
\hline & Propionate & Total SCFA & Propionate & Total SCFA & Propionate & Total SCFA \\
\hline Day 0 & 9.4 & 39.3 & 2.9 & 27.3 & 4.2 & 26.1 \\
\hline Day 1 & 5.4 & 35.2 & 0.0 & 18.5 & 1.8 & 19.1 \\
\hline Day 8 & -1.3 & 19.0 & 0.0 & 9.6 & 10.0 & 27.5 \\
\hline Day 21 & 2.2 & 24.3 & 5.9 & 20.7 & 4.1 & 22.7 \\
\hline
\end{tabular}

Propionic acid and SCFA production were measured in mmol/litre incubation mixture/day.

homogenates in vitro within the first 24 hours of treatment (table 2). The reduction in net total short chain fatty acid production with metronidazole was less pronounced on day 1 , but halved by day 8 . At this stage, in vitro fermentation of stool resulted in virtually no propionate production. By day 21 (14 days after the end of the antibiotic course), propionate production remained at less than $25 \%$ of initial (pre-antibiotic) values and had not returned to the initial values by six weeks. Treatment with erythromycin also suppressed both propionate and total short chain fatty acid production over the course of treatment, but production of both returned to pretreatment values by day 21 . Vancomycin reduced propionate production during the first 24 hours of treatment but by day 8 there was increased production of propionate compared with the pretreatment sample. Total short chain fatty acid production was little affected by vancomycin.

Within 24 hours of starting metronidazole treatment, the plasma propionate concentration fell by $50 \%$ (fig 1 ); by day 8 , it was still $27 \%$ lower than the pretreatment concentration. Plasma propionate concentrations appeared to be increased during treatment with

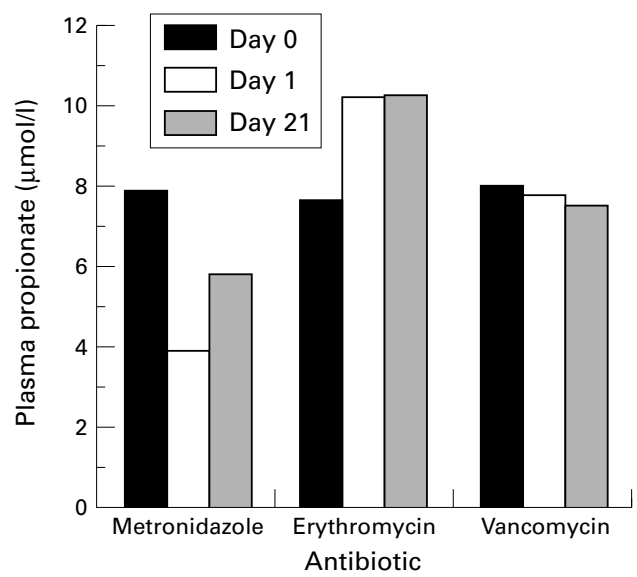

Figure 1 Plasma propionate concentrations during the course of treatment with the three antibiotics. erythromycin but were essentially unchanged by vancomycin. The intermediary metabolites and plasma ammonia concentrations did not show any significant changes during any of the study periods (data not shown).

The child remained clinically well during the study period and did not have any episodes of metabolic decompensation.

\section{Discussion}

Our initial in vitro study demonstrated that all of the antibiotics tested reduced both total short chain fatty acid and propionate production from faecal homogenates. Metronidazole produced the most consistent falls in propionate production at all concentrations studied and with two different polysaccharide sources. These findings are similar to those of Clausen and colleagues who used glucose - a less physiological substrate-in a study of healthy adult volunteers. ${ }^{4}$ Thus, all the antibiotics tested in the in vitro fermentation study had the potential for use in vivo in the patient with propionic acidaemia. However, we chose not to use clindamycin because of the frequent occurrence of antibiotic associated pseudomembranous colitis with this drug. We used a five week period without the administration of antibiotic in each cycle; this has been shown previously to be an adequate period for normalisation of bowel flora in adults. ${ }^{6}$ However, it is possible that this was not adequate for normalisation of bowel flora, and the efficacy of erythromycin might have been underestimated.

Hoverstad et al found that oral clindamycin but not metronidazole reduced total short chain fatty acid concentrations in six healthy volunteers. ${ }^{6}$ The effects of antimicrobial agents on short chain fatty acid concentrations depend on their antimicrobial spectra and colonic concentrations. Although clindamycin was found in high concentrations in the faeces, metronidazole was not detectable. Therefore, the lack of effect of the latter on short chain fatty acid concentrations was attributed to its almost complete absorption in the intestine. However, we found that oral administration of both erythromycin and metronidazole for a week essentially eliminated propionate production when colonic bacteria from stool were incubated with potato starch as substrate. On the other hand, oral vancomycin did not alter the faecal fermentative capacity during or after the course of the treatment. Fermentation products of carbohydrates depend upon the species of anaerobic bacteria as well as their interactions with nutrients. The main propionate producing bacteria include species of bacteroides, peptostreptococcus, succinomonas, and succinivibrio. ${ }^{12}$ Unlike vancomycin and erythromycin, metronidazole is particularly active against these species of anaerobic organisms and, therefore, it is not surprising that metronidazole had such a pronounced effect on propionate production in our study.

Although reduction in gut derived propionate after metronidazole treatment was associated with lowering of the plasma propionate concentration, such an effect was not seen with erythromycin. The plasma propionate 
concentration was used as proxy for changes in whole body production or flux of propionate. The former is influenced by a number of factors including dietary protein intake and catabolism of body proteins and might not be a very sensitive measure of changes in flux. However, it can be measured less invasively than flux, which requires the use of radiolabelled tracers. Hence, a lack of effect on total plasma propionate concentration during erythromycin treatment does not negate its efficacy. Importantly, the suppressive effect of a one week course of metronidazole on faecal fermentative capacity lasts for at least three weeks, and this was reflected in lower plasma propionate concentrations during this period. Interestingly, the patient with methylmalonic acidaemia described by Bain et al showed a continued suppression of faecal propionate concentrations during an intervening 14 day period off treatment. ${ }^{13}$ Thus, it is possible that intermittent courses of metronidazole might be as effective as continuous treatment in reducing propionate production in the gut. We suggest that the efficacy of such an intermittent metronidazole schedule be studied in a larger cohort of children with disorders of propionate metabolism.

Dr Deshpande was supported by the Scientific and Research Committee of Newcastle Health Authority and by the Foundation for the Study of Infant Deaths. Dr Mellon was supported by a grant from the Special Trustees' Fund of the Royal Victoria Infirmary. We wish to thank Dr F Alexander for kindly allowing us to study his patient.
1 Fenton WA, Rosenberg LE. Disorders of propionate and methylmalonate metabolism. In: Scriver CR, Beaudet AL, Sly WS, Valle D, eds. The metabolic and molecular bases of inherited disease, 7th ed. London: McGraw-Hill, 1995:1423-49.

2 Thompson GN, Walter JH, Bresson JL, et al. Sources of propionate in inborn errors of propionate metabolism. Metabolism 1990;39:1133-7.

3 Walter JH, Thompson GN, Leonard JV, Bartlett K, Halliday D. Contribution of amino acid catabolism to propionate production in methylmalonic acidaemia. Lancet 1989;i: $1298-9$

4 Clausen MR, Bonnen H, Tvede M, Mortensen PB. Colonic fermentation to short-chain fatty acids is decreased in antibiotic-associated diarrhea. Gastroenterology 1991;101: $1497-504$.

5 Thompson GN, Chalmers RA, Walter JH, et al. The use of metronidazole in management of methylmalonic and propionic acidaemias. Eur f Pediatr 1990;149:792-6.

6 Hoverstad T, Carlstedt-Duke B, Lingaas E, et al. Influence of ampicillin, clindamycin and metronidazole on faecal excretion of short-chain fatty acids in healthy subjects. Scand F Gastroenterol 1986;21:621-6.

7 Hoverstad T, Carlstedt-Duke B, Lingaas E, et al. Influence of oral intake of seven different antibiotics on faecal shortchain fatty acid excretion in healthy subjects. Scand $f$ Gastroenterol 1986;21:997-1003.

8 Edwards CA, Gibson G, Champ M, et al. In vitro method for quantification of the fermentation of starch by human faecal bacteria. F Sci Food Agric 1996;71:209-17.

9 Mathers JC, Fernandez F, Hill MJ, McCarthy PT, Shearer MJ. Dietary modification of potential vitamin $\mathrm{K}$ supply from enteric bacterial menaquinones in rats. $\mathrm{Br} \mathcal{F}$ Nutr 1990;63:639-52.

10 Remesey C, Demigne C. Determination of volatile fatty acids in plasma after ethanolic extraction. Biochem $\mathcal{F} 1974$; 141:85-91.

11 Harrison J, Hodson AW, Skillen AW, Stappenbeck R, Agius L, Alberti KGMM. Blood glucose, lactate, pyruvate, glycerol, 3-hydroxybutyrate and acetoacetate measurements in man using a centrifugal analyser with a fluorimetric attachment. F Clin Chem Clin Biochem 1988;26:141-6.

12 Miller TL, Wolin MJ. Fermentations by saccharolytic intestinal bacteria. Am f Clin Nutr 1979;32:164-72.

13 Bain $M$, Jones $M$, Borriello $S$, et al. Contribution of gut bacterial metabolism to human metabolic disease. Lancet 1988;i:1078-9. 